\title{
Busca, acesso, compartilhamento e uso de informação em micro e pequenas empresas do Brasil
}

Búsqueda, acceso, compartición y uso de la información en microempresas y pequeñas empresas brasileñas

Seeking, accessing, sharing and using information in Brazilian micro and small enterprises

\section{Luana MAIA WoIDA}

Faculdade de Tecnologia de Garça, Av. Presidente Vargas, 2331 Garça-SP, luanamwoida@yahoo.com.br. Programa de Pós-Graduação em Ciência da Informação (Unesp-Marília)

\begin{abstract}
Resumen
Se investiga la percepción de los empresarios en Brasil respecto a la búsqueda, acceso, intercambio e identificación de necesidades informacionales de forma exploratoria y descriptiva. Los datos fueron recogidos por agentes locales de innovación vinculados al programa estatal ALI de empresarios de 18 ciudades del estado de São Paulo. Los datos sugieren que no hay intercambio de información, que su acceso está limitado a una sola parte de los funcionarios, que no se accede a la información con la frecuencia que se debería, y, si lo hacen, siguen procedimientos informales. Además, la información es muy poco utilizada, especialmente la de los clientes, y la obtenida del entorno exterior no se utiliza para mejorar o innovar los productos, servicios y procesos. Tampoco se documentan los procesos estándares del negocio, lo que complica el acceso a esa información. En conclusión, las PyME no poseen un ambiente informacional sistematizado, lo que sería útil para alcanzar un buen grado de innovación.
\end{abstract}

Palabras clave: Información empresarial. Necesidades de información. Fuentes de información. Uso de información. Brasil. Pequeñas empresas. Microempresas.

\section{Introdução}

A gestão das micro e pequenas empresas (MPE) possui particularidades, pois apresenta escasses de recursos e depende de fatores como a experiência e formação do empreendedor, acesso a recursos e produção de conhecimento. Assim, conseguir desenvolver um produto ou serviço que seja valorizado por um grupo de consumidores é uma tarefa desgastante e repleta de barreiras, entre as quais, destaca-se o acesso à informação confiável e relevante, a qual pode estar disponível em diferentes fontes de informação ou produzida em pesquisas de mercado. No que diz respeito às empresas, trata-se dos subsídios necessários para as atividades da organização, sendo de diferentes tipos: mercado, concorrência, demandas tecnológicas, inovação, sobre clientes e parceiros, canais de distribuição, recursos humanos,

\begin{abstract}
Information search, access, sharing and needs of entrepreneurs of small and medium-sized enterprises in Brazil was researched using exploratory and descriptive methods. The data was gathered by local innovation agents of the government programme LIA from entrepreneurs of 18 São Paulo Estate cities. Results suggest that there is no information sharing, that access to information is restricted to a small group of employees, and that they do not access information as often as they should, and when they do, they follow informal procedures. In addition, information is underutilized, a lot of information from customers is ignored, and information obtained from the external environment is not used to improve or innovate the business, its products, services and processes. Business processes are not recorded systhematically, making them difficult to access. So, SMEs do not have the systematized information environment that is needed to reach a high degree of innovation.
\end{abstract}

Keywords: Business information. Information needs. Information sources. Information use. Brazil. Micro and small enterprises.

financiamentos e, controle de estoques. Assim, informação é um conjunto de dados com significado e contextualizado, usado para melhorar ou construir novos conhecimentos.

Observa-se que o intuito do artigo não contempla uma revisão de literatura extensa sobre estudos de usuários ou sobre o que compõe a competência em informação na Ciência da Informação, uma vez que existe vasta literatura destinada a este escopo, como pode ser observado na proposta de Araújo (2018), ao discutir o que é a Ciência a Informação, percorrendo a formação, os atores, abordagens, as teorias e problemas informacionais. Assim, optou-se por uma literatura que ajudasse a explicar alguns dos conceitos centrais permitindo discutir a informação e o conhecimento do ponto de vista do empresário nos seguintes aspectos extraídos do Questionário de 
Autoavaliação MPE Brasil, usados no Programa Agentes Locais de Inovação do Sebrae: busca, acesso, compartilhamento e identificação de necessidades informacionais. Assim, a pesquisa buscou responder aos seguintes problemas: qual a frequência de compartilhamento de informação nessas empresas? Há busca de informação em fontes externas e junto ao colaborador para identificar oportunidades de inovação? Há busca de informação junto aos clientes? Há informações disponibilizadas (acessíveis) aos colaboradores, visando a tomada de decisão? Há o uso das informações obtidas visando melhorias do desempenho e produtos e processos? Os processos da empresa seguem padrões documentados (informação registra)? Desse modo, o objetivo foi determinado prevalecendo o ponto de vista de que a informação é importante para a gestão das empresas, e mesmo sendo um recurso essencial, pode apresentar falta ou insuficiência de compartilhamento, de acesso e uso.

\section{Metodologia}

Diante do exposto, a presente pesquisa é caracterizada como exploratória e descritiva, usandose média e coeficiente de variação para a apresentação dos resultdos. Os dados foram obtidos em coletas realizadas por vinte Agentes Locais de Inovação do Programa ALI, cuja coleta foi realizada junto aos empresários (as) que fizeram adesão ao Programa ALI entre 2015 e 2016, sendo que as empresas localizavam-se em diferentes cidades do entorno da cidade de Marília$\mathrm{SP}$, Brasil. O diagnóstico no Programa ALI é realizado pela aplicação do Questionário de Autoavaliação MPE Brasil, pelo Radar da Inovação e pela Matriz FOFA. A aplicação do MPE Brasil é uma etapa do Programa ALI, aplicado em cada um dos ciclos do programa, com a função de diagnosticar a maturidade da gestão da empresa, para viabilizar planos de ação sugeridos aos empresários.

\begin{tabular}{|c|c|c|c|c|}
\hline Questões & a & $\mathrm{b}$ & c & $d$ \\
\hline P4. Os dirigentes compartilham informações com os colaboradores? & 0,00 & 0,75 & 1,88 & 2,50 \\
\hline $\begin{array}{l}\text { P6. A busca de informações para identificar oportunidades de inovação incluem as fontes externas } \\
\text { e os colaboradores são incentivados a apresentarem ideias que podem se converter em } \\
\text { inovações? }\end{array}$ & 0,00 & 0,75 & 1,88 & 2,50 \\
\hline $\begin{array}{l}\text { P15. As informações obtidas dos clientes são analisadas e utilizadas para intensificar a sua } \\
\text { fidelidade e captar novos? }\end{array}$ & 0,00 & 0,54 & 1,27 & 1,80 \\
\hline $\begin{array}{l}\text { P19. As informações necessárias para o planejamento, a execução e análise das atividades e } \\
\text { para a tomada de decisão estão definidas e disponibilizadas aos colaboradores? }\end{array}$ & 0,00 & 0,75 & 1,88 & 2,50 \\
\hline $\begin{array}{l}\text { P22. São obtidas e utilizadas informações comparativas na análise do desempenho e melhoria } \\
\text { dos produtos/serviços e processos? }\end{array}$ & 0,00 & 0,45 & 1,13 & 1,50 \\
\hline $\begin{array}{l}\text { P28. Os processos principais do negócio são executados de forma padronizada, com padrões } \\
\text { documentados? }\end{array}$ & 0,00 & 1,20 & 3,00 & 4,00 \\
\hline
\end{tabular}

Tabela I. Questões e sua pontuação

O questionário de Autoavaliação MPE Brasil teve sua construção baseada no Modelo de Excelência da Gestão ${ }^{(M E G)}$ da Fundação Nacional da Qualidade (FNQ), que entre as finalidades pretende ampliar o compartilhamento de informação da empresa, e realizar a auto-avaliação necessária para concorrer ao Prêmio de Competitividade para Micro e Pequenas Empresas. Dessa forma, se constitui de 37 questões, entre as quais algumas sobre o manejo da informação (MPE Brasil, 2014, p. 10). No instrumento MPE Brasil, para cada uma das questões existem quatro alternativas com pontuações crescentes, conforme consta na Tabela I.

Para enfatizar a informação e o conhecimento como subsídios da gestão, foram escolhidas as questões pertencentes à categoria Informação e Conhecimento, incluindo-se ainda as questões 4 , 6,15 e 28 (Sebrae, 2016) de outras categorias.
Justifica-se que uma aproximação com a aplicabilidade prática se deposita em conhecer a realidade de tais empresários para poder, por exemplo, propor um manual de fontes de informação, ou mesmo a projeção de cursos de formação para potencializar o acesso e o compartilhamento de informação no micro e pequeno ambiente dessas organizações, questões que inclusive foram sugeridas para o Sebrae, ao final da investigação. Além disso, em termos práticos, várias intervenções que foram sugeridas e executadas pelos próprios ALIs (agentes locais de inovação) visando mapear e melhorar os processos de gestão em tais empresas. Assim, a metodologia usada para produzir os dados para este artigo foram únicas em termos de coleta, e os resultados obtidos e descritos neste artigo foram usados como parâmetro para a versão do Programa ALI, iniciada em 2019. Assim, a contribuição do artigo está centrada em colocar 
à luz um ambiente com especificidades do ponto de vista da gestão, incluindo o que se compreende pelo manejo da informação, partindo do ponto de vista de que "[...] no hay dos organizaciones iguales, aunque sean del mismo tipo. Cada una tiene sus distinciones, sus personas son diferentes, su cultura, su infraestructura" (Ponjuan-Dante, 2015, p. 61). Em outras palavras, ainda que investigações sobre busca, acesso, compartilhamento e uso sejam frequentes na Ciência da Informação, não existem registros de publicações que contemplem a região de Marília-SP, somadas às empresas de micro e pequeno porte e com a metodologia do Programa ALI, especificamente na etapa de aplicação do questionário MPE Brasil.

\section{Processo informacional}

Desse modo, sabe-se que os processos informacionais são necessários para realizar a gestão do conhecimento e uma parte importante da inteligência organizacional, uma vez que a partir deles torna-se possível tomar decisão e criar conhecimento (Rodriguez-Cruz, 2015, p. 355). No que diz respeito a tais processos informacionais, constam a busca, o acesso, o compartilhamento e o uso da informação. Assim, entende-se que o acesso à informação é o meio ou método usado para localizar um assunto, sendo que o perfil do empresário da micro e pequena empresa pode caracaterizar-se por ser desprovido de conhecimento profundo sobre os tipos de informação, influenciado pela percepção produzida pelo ambiente que o cerca. $\mathrm{O}$ acesso à informação é um dos principais processos para qualquer empresa conhecer produtos, serviços, bem como desenvolver melhorias, compreender e encontrar clientes e demais integrantes necessários à cadeia de entrega de valor ao consumidor. Dessa forma, no que concerne ao contexto das MPEs da região da Alta Paulista, interior de São Paulo, é importante compreender como é conduzido o acesso à informação, considerando-se que para isso, dependa do conhecimento das fontes importantes para o negócio da empresa.

As fontes são o local no qual podem ser encontradas as informações relevantes, cujos tipos são descritos em Ponjuán Dante (2007, p. 24) e em Martín Vega (2014, p. 23-44). As fontes informais externas, costumam advir da interação entre pessoas, talvez constituindo-se como uma das mais usadas pelos empresários de negócios de micro e pequeno porte devido à experiência e infraestrutura disponíveis.

A busca por informação, intensificada a partir da Década de 90 devido ao uso de bases de dados (Moncada-Hernández, 2014), é para Marchionini (1995, p. 28) "[...] o processo dirigido para as necessidades humanas de informação para que possam integir com o ambiente", bem como são ações executadas pelas pessoas ao tentar satisfazer suas necessidades de informação, as quais podem ser intencionais (Olmeda Gómez et al, 2014 , p. 193). A qualidade e o acesso às fontes pode facilitar ou dificultar essa busca, bem como outras variáveis como as barreiras, como as psicológicas, as demográficas e os papéis (Olmeda Gómez et al., 2014). Choo (2003) explica que as necessidades de informação emergem em situações específicas e Dorner, Gorman e Calvert (2015, p. 30-31) argumentam que existem necessidades não expressas, que podem resultar em obstáculos ou em inércia comportamental, cujo resultado é não se converter em oportunidade de busca e de construção de conhecimento para soluções no ambiente empreendedor, o que ajuda a explicar alguns dos impedimentos dos processos informacionais.

O uso da informação acessada também é outro aspecto relevante, uma vez que uma parte dessas informações acessíveis acaba não sendo incluída na gestão do negócio. Os motivos para isso apontam para a falta de preparo para identificar as informações importantes. Isto é, mesmo com acesso à informação, falta ao empresário preparo para perceber quando uma informação pode ser útil. O uso integrado de diferentes informações advindas do conhecimento tácito, do explícito e do cultural, resulta em grupos com práticas de compartilhamento de informação e de significados sobre o ambiente laboral (Ponjuán Dante, 2007, p.116), propiciando com que melhorias nos processo de gestão e de manejo da informação sejam incluídos de maneira concreta nas práticas da organização. Dessa forma, espera-se que o uso da informação alcance como resultado uma mudança no conhecimento (Choo, 2003, p. 107). O uso da informação, quando eficiente, resulta no comportamento adaptativo, demonstrando reação ao ambiente (Choo, 2003, p. 404). As diferentes etapas do manejo da informação, como em um ciclo, são percebidas na gestão do conhecimento e na gestão da informação, importantes bases para a inteligência organizacional, como sugere PonjuánDante (2015, p. 66):

Inteligencia organizacional es la capacidad de una organización de emplear con eficacia y eficiencia sus recursos tangibles e intangibles en el logro de sus objetivos estratégicos, utilizando asertivamente las técnicas y herramientas de la gestión, potenciando la filosofía de calidad total y asegurando un permanente estudio y posible renovación de sus procesos organizacionales. 


\section{Resultados e discussão}

Diferentes medidas estatísticas foram usadas para a descrição e construção de inferências, sendo que neste artigo é exposto apenas a média e o CV das questões por cidade:

O coeficiente de variação é uma medida usada para caracterizar a dispersão ou variabilidade dos dados, realizando a razão entre o desvio padrão e sua média, sendo representado em porcentagem pelo motivo de que o resultado é justamente o peso do desvio padrão em relação à distribuição dos dados, em outras palavras, se trata de identificar quanto os desvios atingem em relação à média percentual.

Foram analisadas 1424 empresas em 17 cidades no entorno de Marília-SP. As empresas são subdivididas por setor. Sendo que o Comércio soma 548 empresas, a Indústria 306 e os Serviços com 570 , totalizando 1424 . As distribuições das empresas por segmento indicam que no setor comércio o destaque é para o segmento vestuário, com 164 empresas. Referente ao setor indústria, identificou-se que existem mais empresas dos segmentos alimentos e bebidas com 39 empresas e minimercado somando também 39 empresas. Por sua vez, o setor serviços destaca o segmento alimentação fora do lar, totalizando 200 empresas. A Tabela II apresenta as medidas resumo (média e coeficiente de variação) para cada uma das questões do MPE Brasil escolhidas, porém por cidade.

\begin{tabular}{lcccccc}
\hline Cidade & P4 & P6 & P15 & P19 & P22 & P28 \\
\hline Média (CV) & Média (CV) & Média (CV) & Média (CV) & Média (CV) & Média (CV) \\
\hline Ocauçu & $2,19(46 \%)$ & $0,75(0 \%)$ & $1,27(0 \%)$ & $0,45(0 \%)$ & $1,13(0 \%)$ & $1,20(0 \%)$ \\
\hline Vera Cruz & $0,00(0 \%)$ & $0,75(0 \%)$ & $0,54(0 \%)$ & $0,45(0 \%)$ & $0,45(0 \%)$ & $1,20(0 \%)$ \\
\hline Herculândia & $0,00(0 \%)$ & $0,75(0 \%)$ & $0,54(0 \%)$ & $0,45(0 \%)$ & $1,13(0 \%)$ & $1,20(0 \%)$ \\
\hline Jafa & $0,75(0 \%)$ & $0,75(0 \%)$ & $0,54(0 \%)$ & $0,45(0 \%)$ & $0,90(43 \%)$ & $0,80(86 \%)$ \\
\hline lacri & $0,50(86 \%)$ & $0,5(86 \%)$ & $0,54(0 \%)$ & $0,15(173 \%)$ & $1,13(0 \%)$ & $0,00(0 \%)$ \\
\hline Oriente & $0,45(91 \%)$ & $0,45(91 \%)$ & $0,58(127 \%)$ & $0,27(92 \%)$ & $0,72(51 \%)$ & $1,20(0 \%)$ \\
\hline Parapuã & $1,02(77 \%)$ & $0,56(62 \%)$ & $0,54(0 \%)$ & $0,66(82 \%)$ & $0,30(176 \%)$ & $0,82(128 \%)$ \\
\hline Chavantes & $1,33(62 \%)$ & $0,66(41 \%)$ & $0,56(61 \%)$ & $0,39(41 \%)$ & $0,79(46 \%)$ & $1,05(40 \%)$ \\
\hline Bastos & $1,29(64 \%)$ & $0,89(76 \%)$ & $0,74(74 \%)$ & $0,47(85 \%)$ & $0,63(67 \%)$ & $1,48(69 \%)$ \\
\hline Pompéia & $1,24(69 \%)$ & $0,74(36 \%)$ & $0,72(49 \%)$ & $0,35(83 \%)$ & $0,57(56 \%)$ & $1,40(53 \%)$ \\
\hline Garça & $1,10(79 \%)$ & $0,89(70 \%)$ & $0,63(79 \%)$ & $0,61(67 \%)$ & $0,65(81 \%)$ & $1,53(64 \%)$ \\
\hline S. C. do Rio Pardo & $1,19(66 \%)$ & $1,01(65 \%)$ & $0,80(65 \%)$ & $0,45(102 \%)$ & $0,55(84 \%)$ & $1,55(75 \%)$ \\
\hline Assis & $1,23(62 \%)$ & $0,92(56 \%)$ & $0,61(64 \%)$ & $0,62(66 \%)$ & $0,64(69 \%)$ & $1,60(62 \%)$ \\
\hline Ourinhos & $1,47(68 \%)$ & $0,73(64 \%)$ & $1,06(68 \%)$ & $0,35(120 \%)$ & $0,77(66 \%)$ & $1,49(82 \%)$ \\
\hline Tupã & $1,26(59 \%)$ & $0,82(49 \%)$ & $0,81(64 \%)$ & $0,43(79 \%)$ & $0,77(51 \%)$ & $1,47(67 \%)$ \\
\hline Marília & $1,19(67 \%)$ & $0,87(68 \%)$ & $0,68(72 \%)$ & $0,49(67 \%)$ & $0,70(69 \%)$ & $1,56(71 \%)$ \\
\hline Geral & $1,21(68 \%)$ & $0,86(65 \%)$ & $0,73(72 \%)$ & $0,49(78 \%)$ & $0,70(65 \%)$ & $1,53(71 \%)$ \\
\hline
\end{tabular}

Tabela II. Medidas resumo por questão e cidade

Assim, a questão P4, que se refere ao compartilhamento de informações dos dirigentes com os colaboradores, obteve média geral de 1,21, tal valor está mais próximo à alternativa $b$, que significa que o compartilhamento das informações com os colaboradores ocorre esporadicamente, no entanto, o coeficiente de variação foi de $68 \%$ o que caracteriza dispersão moderada das respostas, ou seja, as empresas responderam alternativas distintas, portanto, sem consenso. Nesse caso, o compartilhamento é realizado sem a devida atenção por parte das empresas, sendo possível que enfrentem problemas para a difusão da informação (Cunha y Cavalcanti, 2008), mas também dificuldades que recaem sobre o processo de aprendizado, da recuperação da informação e da criação de mais informação (Choo,
2003). Além disso, na medida em que o ato de compartilhar possui barreiras, isso refletirá no uso da informação. Nesse caso, o ato de compartilhar advém da instrução e de estímulos que deveriam vir dos dirigentes, mas encontram-se medidas restritivas e possivelmente a ausência de uma cultura informacional, posto que nem todos os dirigentes compartilham informação.

A questão P6 refería-se a busca de informações para identificar oportunidades de inovação que incluíssem as fontes externas e os colaboradores, sendo que estes deveriam ser incentivados a apresentarem ideias que podem ser convertidas em inovações. Obteve-se média geral de 0,86 e $\mathrm{CV}=65 \%$, o valor médio se aproximou da alternativa $b$, a qual significava que as informações e conhecimentos para identificar oportunidades de 
inovação são obtidos eventualmente nos relacionamentos externos e internamente os colaboradores são incentivados informalmente a apresentarem ideias que podem converter-se em inovações. Entretanto, a dispersão das respostas foi moderada. Desse modo, infere-se que tanto a busca parece explorar de maneira insuficiente os stakholders como fontes de informação, como também os próprios funcionários parecem ser incentivados a compartilhar suas ideias, porém de maneira não sistematizada, revelando uma provável falta de planejamento e inserção nas tarefas formais. Essas informações revelam que o engajamento em localizar informações (Marchionini, 1995) é inadequado, prejudicando o acesso à informação e interferindo diretamente na integração das informações. Uma busca insuficiente também demonstra improviso e talvez incerteza sobre o que realmente é considerado importante em termos de informação para essas empresas. Uma busca sistematizada indica uma organização bem estruturada entorno do fluxo informacional, do qual retira subsídios para a decisão e para a inovação. $O$ que parece não ser o caso das empresas que participaram do MPE Brasil aplicado no Programa ALI 2015/2017.

A questão P15 buscou identificar se as informações obtidas dos clientes são analisadas e utilizadas para intensificar a sua fidelidade e captar novos. Encontrou-se a média geral de 0,73 e CV $=72 \%$, cujo valor médio está mais próximo à alternativa b, ou seja, as informações obtidas são analisadas ocasionalmente para fidelizar os clientes atuais, no entanto, a dispersão das respostas foi alta. Assim, ainda que parte das informações seja analisada pelas empresas visando fidelizar e captar novos clientes, há indicativo de que uma parcela dessas empresas parece não executar essa verificação. Assim, ao que parece, informações dessa natureza não deveriam ser menosprezadas, uma vez que auxiliam a empresa a obter um comportamento adaptativo, conforme sustenta Choo (2003).

A questão P19 buscava verificar se as informações necessárias para o planejamento, a execução, a análise das atividades e para a tomada de decisão estavam definidas e disponibilizadas aos colaboradores. A média geral foi de 0,49 e CV $=78 \%$, o valor médio aproxima-se da alternativa $b$, a qual se concentrava em indicar que algumas informações para o planejamento, análise e execução das atividades para a tomada de decisão são definidas e disponibilizadas. No entanto, a dispersão das respostas foi alta. Nota-se nesta questão que podem haver problemas envolvendo a difusão de informações nas empresas, uma vez que apenas algumas informações são realmente definidas e disponibilizadas. A disseminação da informação é necessária para alavancar o processo de aprendizagem, bem como para facilitar a recuperação da informação e a criação de mais informações advindas do que é acessado pelo compartilhamento (Choo, 2003). Por outro lado, um compartilhamento ineficiente impossibilita $o$ uso das informações para acompanhar as mudanças do ambiente e para tomar decisões (Choo, 2003). Nesse sentido, é necessário questionar o que conduz à não disponibilização de informação. Aqui cabem algumas considerações: as empresas podem submeter os colaboradores à escassez de informação acreditando que esse isolamento da informação pode não afetar os resultados do trabalho; as empresas podem desconhecer as necessidades informacionais dos colaboradores, uma vez que não são expressas, e, com isso, produzir a inércia comportamental (Dorner, Gorman y Calvert, 2015).

A questão P22 visava verificar se as informações são obtidas e utilizadas comparativamente na análise do desempenho e melhoria dos produtos/serviços e processos. A média obtida foi de 0,70 e o $\mathrm{CV}=65 \%$, cujo valor médio se aproximou da alternativa $b$, que significa que são obtidas informações comparativas externas, mas não são utilizadas na análise do desempenho e melhoria dos produtos/serviços e processos, considerando que a dispersão das respostas foi moderada. Há nítida falta de atenção com relação às informações disponíveis em fontes externas. Parece haver falta de percepção sobre a inserção da empresa em um ambiente dinâmico. Por outro lado, a verificação de informações para tal propósito também pode ser prejudicada em virtude de não existir a identificação de necessidades, conduzindo à uma busca de informação limitada, como sugere Choo (2003, p.405). O mais evidente é que inexiste uma sistematização do fluxo de informação para uso estratégico por parte de muitas das empresas que participaram da pesquisa, sugerindo pouco engajamento para mudar, melhorar ou apronfudar o que conhececem.

Verifica-se que a questão P28, que se refere aos processos principais do negócio serem executados de forma padronizada, com padrões documentados, obteve média 1,53 , valor mais próximo $a$ alternativa $b$, o que significa que os processos principais do negócio são executados de forma padronizada, mas os padrões não são documentados, cujo coeficiente de variação foi de $71 \%$, com variabilidade das respostas alta nesta questão. A falta de consenso sobre as respostas indica que processos padronizados, se é que existem, estão pouco presentes como prática de documentação nas empresas. O registro da informação é necessário para obter a recuperação, a disseminação e controlar o acesso quando necessário, 
considerando ainda que a documentação facilitaria a integração entre informações (Choo, 2003) e poderia dar maior foco a problemas específicos. Contudo, há que se considerar que ainda que ocorressem os registros das informações, seria necessário organizar tais informações para se tornarem acessíveis, talvez um pouco distante da realidade das micro e pequenas empresas.

\section{Considerações finais}

Sobre a busca, o acesso, o compartilhamento, a identificação de necessidades verificou-se um contexto bastante improvável para se realizar a gestão da informação, a gestão do conhecimento e a inteligência organizacional, uma vez que os processos informacionais básicos ainda são incipientes, pois nem todos os dirigentes compartilham informação com os colaboradores, diminuinto a disponibilização de informação aos funcionários. Assim, estes não acessam a informação com frequência ou na quantidade que deveriam para planejar, executar, analisar e tomar decisão, bem como para interferir na inovação desejada pelo Programa ALI. O compartilhamento, quando ocorre, é efetivado em meios informais, sem que uma sistematização conduza ao fluxo de informação melhor controlado ou previsto. O problema não reside na informalidade, o que não seria incomum em empresas desse porte, reside sim na falta de inserção das fases do ciclo informacional nas tarefas rotineiras. Parte dos resultados sugere problemas quanto ao uso da informação, pois há subutilização das obtidas junto aos clientes.

Também foi possível verificar uma ambiguidade à respeito do tratamento dado à informação: por um lado informações são obtidas externamente e por outro, não são usadas para melhorar produtos e serviços indicando provável coleta ineficiente, falta de identificação de necessidades, ou mesmo, uma falta de conhecimento sobre a qualidade das fontes de informação e com que tipo de informação cada fonte poderia contribuir.

Por fim, percebeu-se que o fato dos padrões não serem documentados indica que conhecimentos importantes podem não estar sendo registrados, inviabilizando acesso a eles. Os prejuízos para isso incluem a impossibilidade de converter informação em conhecimento e de promover condições para gestão organizacional. Nesse sentido, o objetivo do presente trabalho foi alcançado revelando que as MPEs não possuem um ambiente informacional com favorabilidade para inovar, situação que merece atenção, uma vez que o intuito do Programa ALI é melhorar a gestão e também a inovação em empresas desse porte em todo o território brasileiro.

\section{Notas}

Este trabalho foi financiado com Bolsa de Pesquisa CNPq EXP-SA entre 2016 e 2017, para no Programa ALI do SEBRAE voltado à inovação.

\section{Referencias}

Araújo, C. A. A. (2018). O que é ciência da informação. Belo Horizonte: KMA.

Choo, C. W. (2003). A organização do conhecimento. Como as organizações usam a informação para criar significado, construir conhecimento e tomar decisões. São Paulo: Editora Senac São Paulo.

Cunha, M. B. da; Cavalcanti, C. R. de O. (2008). Dicionário de Biblioteconomia e Arquivologia. Brasília: Briquet de Lemos.

Dorner, D. G.; Gorman, G.E.; Calvert, P. J. (2015). Information needs analysis: principles and practice in information organizations. Londres: Facet Publishing, 2015.

Marchionini, Gary (1995). Information seeking in electronic environment. New York: Cambridge University Press.

Marcial, E. C.; Grumbach, R. J. dos S. (2005). Cenários prospectivos: como construir um futuro melhor. 3.ed. Rio de Janeiro: FGV. (Coleção FGV Negócios)

Martín Vega, A. (2014). Las fuentes de información. In: Departamento de Biblioteconomía y Documentación. Técnicas de búsqueda y uso de la información. Madrid: Editorial Universitária Ramon Areces. 23-56.

Moncada-Hernández, S. G. (2014). Cómo realizar una búsqueda de información eficiente. Foco en estudiantes, profesores e investigadores em el área edicativa. // Investigación em Educación Médica. 3:10, 106-115.

Olmeda Gómez, C. (2014). La búsqueda de información em entornos electrónicos. // Departamento de Biblioteconomía y Documentación. Técnicas de búsqueda y uso de la información. Madrid: Editorial Univ. Ramon Areces. 190-212.

Ponjuán Dante, G. (2007). Gestion de información. Dimensiones e implementaçción para el éxito organizacional. Madrid: TREA.

Ponjuán-Dante, Glória. (2015). Inteligencia Organizacional, Gestión de Información, Gestión del Conocimiento: Vinculos y Complementariedad. In: Valentim, Marta Lígia Pomim; Más-Basnuevo, Anays (orgs.) (2015). Marília: Oficina Universitária; São Paulo: Cultura Acadêmica.

Rodríguez-Cruz, Yunier (2015). La Inteligencia Organizacional Bajo un Enfoque Integrador de Gestión Documental, de Información y del Conocimiento. // Valentim, Marta Lígia Pomim; Más-Basnuevo, Anays (orgs.) (2015). Marília: Oficina Universitária; São Paulo: Cultura Acadêmica,.

Serviço Brasileiro de Apoio às Micro e Pequenas Empresas. (2016). Questionário de Autoavaliação MPE Brasil: Prêmio de competitividade para micro e pequenas empresas. http:/www.mbc.org.br/mpe/wp-content/uploads/2016/04/5Question\%C3\%A1rio_MPE_2016_IMP.pdf (23-03-2017).

Serviço Brasileiro de Apoio às Micro e Pequenas Empresas. (2017). Prêmio MPE Brasil. 2017. http://www.sebrae. com.br/sites/PortalSebrae/ufs/ba/premios/premio-mpe-br asil,43225f1151287410VgnVCM1000003b74010aRCRD (20-09-2017).

Tarapanoff, K. (2006). Informação, Conhecimento e Inteligência em Corporações: relações e complementariedade. // Tarapanoff, K. (Org.). Inteligência, informação e conhecimento em corporações. Brasília: IBICT, UNESCO. 19-35.

Enviado: 2018-04-18. Segunda versión: 2019-05-30 Aceptado: 2019-06-06. 